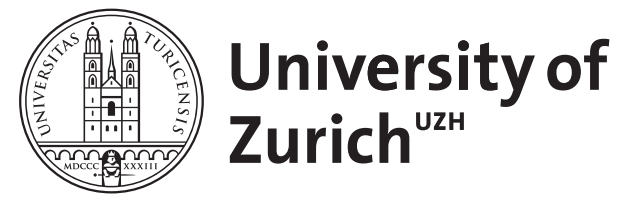

Severe extrapyramidal syndrome after exposition to isoamyl acetate vapour

Baumann, C R ; Jenni, R ; Jung, H H

DOI: https://doi.org/10.1007/s00415-008-0800-3

Posted at the Zurich Open Repository and Archive, University of Zurich ZORA URL: https://doi.org/10.5167/uzh-10050

Journal Article

Published Version

Originally published at:

Baumann, C R; Jenni, R; Jung, H H (2008). Severe extrapyramidal syndrome after exposition to isoamyl acetate vapour. Journal of Neurology, 255(5):762-763.

DOI: https://doi.org/10.1007/s00415-008-0800-3 
Christian R. Baumann

René Jenni

Hans H. Jung

\section{Severe extrapyramidal syndrome after exposi- tion to isoamyl acetate vapour}

Received: 2 July 2007

Received in revised form:

25 September 2007

Accepted: 29 October 2007

Published online: 17 March 2008

Sirs: At room temperature, the lowmolecular-weight ester isoamyl acetate (IAA, syn. 3-methyl-1butanol acetate, $\mathrm{C}_{7} \mathrm{H}_{14} \mathrm{O}_{2}$ ) is a liquid with an intense, but pleasant banana-like odour (Fig.1). Honey bees use IAA as an alarm pheromone, i.e. a chemical substance produced by living organisms that transmits a message to other members of the same species. Bees store IAA in their poison sting. After release in the course of an attack, the odour of IAA alerts other bees to behave defensively, i.e. sting or charge [1]. Due to its strong smell, which can be perceived already at low concentrations, and because of supposedly low toxicity, IAA is used to test the effectiveness of respirators or gas masks. IAA vapour is known to irritate eyes, skin and respiratory tract, and to cause mild

C. R. Baumann, MD (区) • H. H. Jung, MD

Dept. of Neurology

University Hospital

Frauenklinikstrasse 26

8091 Zürich, Switzerland

Tel.: 01-255/5511

Fax: 01-255/4380

E-Mail: cbaumann@bidmc.harvard.edu

R. Jenni, $P h D$

Swiss Federal Institute of Technology

Zurich, Switzerland

C. R. Baumann, MD

$\stackrel{\circ}{\sim}$ Harvard Institutes of Medicine

$z$ Beth Israel Deaconess Medical Center

B Boston, USA

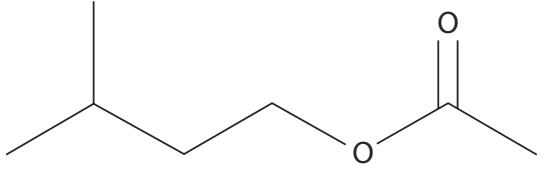

Fig. 1 Chemical structure of isoamyl acetate

unspecific central nervous system symptoms [2]. Herein, we present a patient who developed a severe reversible extrapyramidal syndrome after inhalation of IAA.

Together with his troop, a 21year-old soldier was ordered to test the impermeability of his gas mask with IAA gas. In Switzerland, this procedure is called "banana gas test". In the days prior to the test, he had the same diet as his army colleagues. He did not suffer from any kind of injury during the few weeks before the test, and renewal of tetanus vaccination was several months earlier. Furthermore, he did not consume drugs, and was not on any medication prior to the test. During the "banana gas test", our patient remarked a discrete banana-like odor. Half an hour later, dizziness with disturbed shimmering vision occurred, together with severe pain and stiffness in his jaw, and a progressive difficulty to swallow. The military physician consulted assigned the patient immediately to the emergency unit of our hospital. On neurological examination, we found signs of a cholinergic crisis, including bilateral miosis, hypersalivation, increased sweating, and nausea. Furthermore, the patient showed an extrapyramidal syndrome, including severe painful rigidity of the mimic muscles, particularly in the jaw, but also in his neck and arms, consecutively leading to generalized slowness of motor functions, severe dysarthria, and trismus with inability to close eyes and mouth. Furthermore, we found a high-frequency medial amplitude tremor in both arms, injection of both conjunctivae, and an exanthema on face and trunk. Cranial nerve findings, tendon reflexes, and sensibility were normal. Blood pressure was 130/65 mm Hg in lying position, heart rate was 64 per minute. After intravenous administration of $5 \mathrm{mg}$ biperiden, muscle tone and movements partially normalized. The patient reported feeling a subjective relaxation. Later on, tremor amplitude and frequency also decreased.

Laboratory studies showed no evidence of intoxication or infection. Potassium was discretely decreased (3.2 mmol/l, normal: 3.55.0 ), creatinine kinase was elevated (819 U/l, normal: <190), leukocyte count slightly increased $\left(10.68 \times 10^{3} / \mu \mathrm{l}\right)$. Other serum electrolytes and liver/renal function tests were normal. Toxicological screening analysis did not reveal intoxication by drugs or pharmaceuticals. Cerebral computed tomography was normal.

During the following 48 hours, the neurological symptoms remitted without further medication, whereas a very discrete erythematous exanthema remained on the right forearm.

After inhalation of IAA vapour, our patient developed a cholinergic-like crisis with a severe extrapyramidal syndrome, which remitted after anticholinergic treatment with biperiden. The skin findings in our patient were interpreted as a toxic-irritative exanthema, probably due to the exposition to IAA. It is known that IAA vapour may cause central nervous system symptoms such as headache, nausea, dizziness, drowsiness, disturbed coordination and confusion [2]. However, to our best knowledge, an acute cholinergic-like crisis with severe extrapyramidal symptoms as a consequence of isoamyl acetate inhalation has not been reported before. Only in an old study dating from 1933 was 
spastic paralysis observed in guinea pigs subsequent to subcutaneous application of IAA [3].

Based on history, clinical findings, the course of the symptomatology, laboratory examinations, and cranial computed tomography, we could not find other potential causes for the symptomatology, such as other intoxication, adverse reactions to other medications, botulism, tetanus, hypocalcemia, meningitis, or encephalitis. Hence, the observations in our patient suggest that IAA may enhance cholinergic functions and inhibit the nigrostriatal dopaminergic system via unknown mechanisms. Pertaining to the 'fight or flight' reaction of honey bees after exposition to IAA, Hunt raised the hypothesis whether IAA might stimulate the release of neuropeptides and/or biogenic amines [1]. There might be an analogous situation as found in organophosphate poisoning where inhibition of synaptic acetylcholinesterase may lead to acute cholinergic crisis, and neurotoxic extrapyramidal symptoms have also been observed and considered to be related to the impediment of acetylcholine to modify the dopaminergic system [4].

In conclusion, this case documents a cholinergic-like crisis with severe extrapyramidal syndrome after inhalation of isoamyl acetate vapour in a military camp, but the underlying pathophysiology remains hypothetical. Based on our observations, we believe it is not acceptable to expose young soldiers to a substance with unclear toxicity.

\section{References}

1. Hunt GJ (2007) Flight and fight: A comparative view of the neurophysiology and genetics of honey bee defensive behavior. J Insect Physiol 53:399-410

2. National Library of Medicine, see: http://chem.sis.nlm.nih.gov/ chemidplus/jsp/common/ChemFull. jsp?MW $=130.186$

3. Flury F, Wirth W (1933) Zur Toxikologie der Lösungsmittel. Archiv fuer Gewerbepathologie und Gewerbehygiene (today: International Archives of Occupational and Environmental Health) 5:1-90

4. Hsieh BH, Deng JF, Ger J, Tsai WJ (2001) Acetylcholinesterase inhibition and the extrapyramidal syndrome: a review of the neurotoxicity of organophosphate. Neurotoxicology 22:423-427 